\title{
The relationship between Contact Resistance and Roughness (Sq) of a Bi-layered Surface using a Finite Element Model
}

\author{
J.W.McBride \\ Dept of Mechanical Engineering \\ University of Southampton Southampton, UK \\ jwm@soton.ac.uk
}

\author{
H.Liu \\ Dept of Mechanical Engineering \\ Jiangsu University \\ hong_liu@ujs.edu.cn
}

\begin{abstract}
The application of a structured and rough surface, and the effect the roughness has on contact resistance is considered in an electrical contact application. The surfaces are designed to enhance roughness, with an amplitude parameter (Sq, RMS) of 0.1-1 $\mu \mathrm{m}$. An established finite element model of a bi-layered rough contact surface is extended to include the pre-processing and analysis of a model rough surface. The application is a gold-coated multi-walled carbon nanotube composite $(\mathrm{Au} / \mathrm{CNT})$, designed as a solution for electrical contacts in low current switching applications. To determine the contact resistance for these surfaces, a preliminary step is required to determine the effective resistivity.
\end{abstract}

Model surfaces are compared to a measured rough surface, where the sample length of the surface and the sensor interaction with the measured surface are identified as key parameters. The finite element model provides an output of the interactions between a hemi-spherical ball and the rough $\mathrm{Au} / \mathrm{CNT}$ surface, with the results shown as a map of the points of contact as a function of the applied force $(\mathrm{mN})$. An automated method of post processing the image data is used to determine the number of contact points and the best fitting radius of the contact area. It is shown that the contact resistance increases with surface roughness $(S q)$.

Keywords-Rough surfaces, contact resistance, effective resistivity, composite surfaces.

\section{INTRODUCTION}

The application of a structured and rough surface and the effect roughness has on contact resistance is considered in an electrical contact application. The surfaces are computationally generated models, designed to enhance roughness, with an amplitude parameter (RMS) typically of 0.1-1 $\mu \mathrm{m}$. The surfaces are integrated into an established Finite Element Analysis (FEA) model of a rough surface in contact with a hemispherical smooth surface. The model application is focused on a gold-coated multi-walled carbon nanotube composite ( $\mathrm{Au} / \mathrm{CNT})$, designed as a solution for electrical contacts in low current switching applications [13]. The work is however more generally applicable to rough contact surfaces.

In previous studies, applied to the Au/CNT application, a FEA contact model has been developed [4-8] and verified. Fig. 1 is a typical example of the measured rough surface, while Fig. 2 is a SEM image of a typical surface. The data shown in Fig.1\&2 are for a composite surface of $50 \mu \mathrm{m}$ high, vertically aligned, carbon nanotubes partially shown in Fig.2 and coated with a $500 \mathrm{~nm}$ nominal layer of Au (referred to as a 505 surface) [8,9]. The measured rough surface is shown over a measured area of $0.15 \mathrm{~mm} \times 0.15 \mathrm{~mm}$ with a sample length of $(\delta) 0.25 \mu \mathrm{m}$.

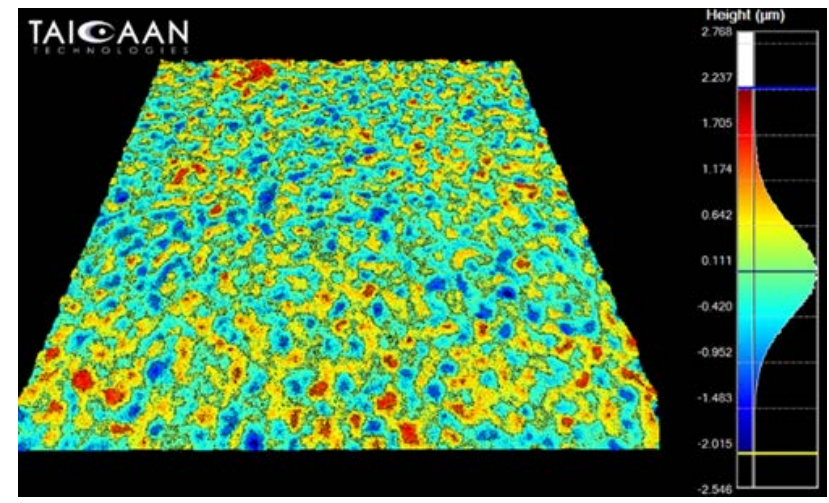

Fig.1 XYRIS 2020L 0.15x0.15 mm, $\delta=0.25 \mu \mathrm{m}, S q=0.528 \mu \mathrm{m}$

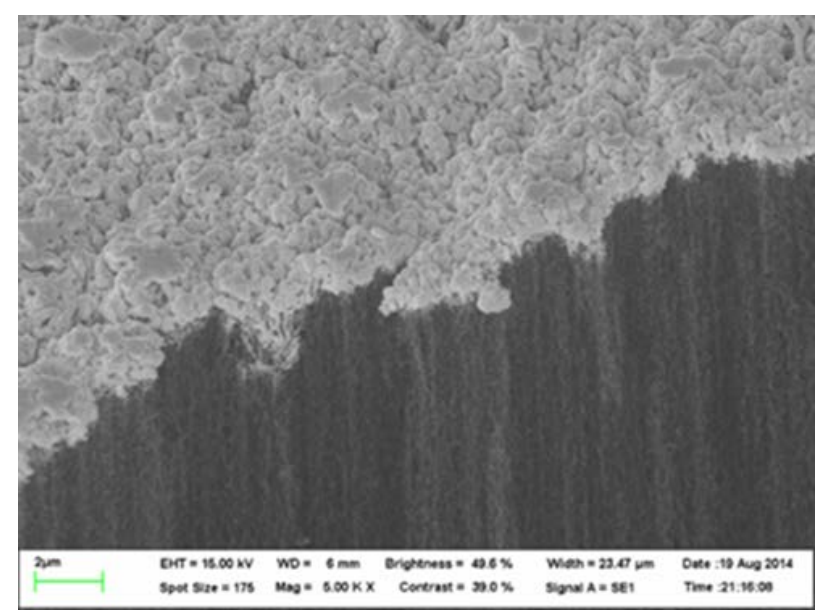

Fig. 2 SEM data, over approximately $20 \mu \mathrm{m}$ of the surface

The initial FEA studies [4-5] were used to compare the loading and unloading characteristics for a range of forces $(0.25-2 \mathrm{mN})$ based on reference data for Hardness and Elasticity determined from nano-indentation tests with a 200 $\mu \mathrm{m}$ radius probe; on a smooth 505 surface in [4], and for a measured rough surface in [5]. In [6] the FEA model was extended and results compared to contact resistance data from a $1 \mathrm{~mm}$ hemispherical ball used in a nano-indentation test, for a smooth 505 surface. The work showed the importance of the effective resistivity. In [7] the work is [6] was extended to a measured rough surface and linked to contact resistance measurements. It was shown that effective resistivity was not constant for a given surface and the 
resulting contact resistance also depended on the location of the ball on the measured surface. The variation was explained to be linked to the waviness of the measured surface. This result explained the variability observed in the initial measurements used to determine the surface Hardness and Elasticity, [4-5]. In [8], a computationally generated model surface was used to investigate the relationship with contact area. The model surface was designed to replicate both the local roughness and the waviness of the measured surface. In this paper we extend the work in [8], by including the contact resistance calculation and investigate the change in the surface roughness, by constraining the waviness issues identified in [7] and [8].

As already noted, a fundamental parameter for the calculation of the contact resistance (or in this case the constriction resistance as there is no significant contribution from film contamination or oxide film formation); is the effective resistivity of the surfaces. It was shown in [9], based on the modified nano-indentation test used in [6], for a $1 \mathrm{~mm}$ ball; that the effective resistivity was a function of the composite mixture, and related to a ratio of the metallic film thickness over the CNT growth height. Further to this it was observed that at higher force levels $(>1.2 \mathrm{mN})$, the effective resistivity increased for a given surface (e.g. 505), as the probe used extended further into the surface. This was not fully described previously but is linked to conformity of the $\mathrm{Au}$ upper surface of the $\mathrm{Au} / \mathrm{CNT}$, (see discussion below and Fig. 5). In [9] the effective resistivity $\left(\rho_{e}\right)$, was determined from the measured contact resistance $\left(R_{c}\right)$ using Eq. (1).

$$
\rho_{e}=2 R_{c} \sqrt{\frac{F}{H \pi}}
$$

The use of Eq.1 assumes a single contact point with a circular contact area. In a rough surface as used in the experiments the area of contact will be distributed over a wider area and constrained by the cluster radius as described in Eq.2, [10]. The effective resistivity as determined from Eq.1, is only an approximation, and is not easily relatable to a sheet resistance as it includes the measured resistance of the interaction with the $1 \mathrm{~mm} \mathrm{Au}$ coated Stainless-Steel hemi-spherical surface, shown in Fig.4. It is also noted that by using Eq.1, we do not account for thin film conduction processes. In this work, as in [7], we determine the constriction resistance from the contact area of the rough surface generated by the FEA (ANSYS v18.1) model, with $N$, the number of contact spots (a-spots), $a$ the mean radius of the a-spots and $r$ the Holm radius (or cluster radius), using the Greenwood relationship [8], Eq (2).

$$
R_{C}=\frac{\rho_{e}}{2 N a}+\frac{\rho_{e}}{2 r}
$$

This relationship is determined from the summation of the constriction of current flow through the cluster radius, in series with the number of smaller constrictions associated with the distributed contact spots. It does not account for the interaction of electron flow, between the contact spots, as discussed in [8].

The FEA model [4-8] has been developed using the mechanical characteristics of a rough surface and does not include a multi-physics approach for the determination of the constriction resistance, due to convergence issues associated with the complexity of the model.

\section{SuMmary OF PREVIOUS STUDIES AND REFERENCE DATA}

\section{A. A bi-layyered surface in contact with a $1 \mathrm{~mm}$ hemi- spherical surface.}

An FEA mesh of the interaction of the Au coated ball in contact with a bi-layered surface is shown in Fig.3. The window in the image is a close up of the contact region and shows the detail of the mesh on the upper Au coated surface of the bi-layer. The computational methods used are detailed in [4-8]. The upper hemisphere is Au coated, stainless steel but modelled as a solid Au surface and the lower surface characterized by an upper surface defined as a mixture of $\mathrm{Au}$ and CNT and a lower surface of CNT only, [6].

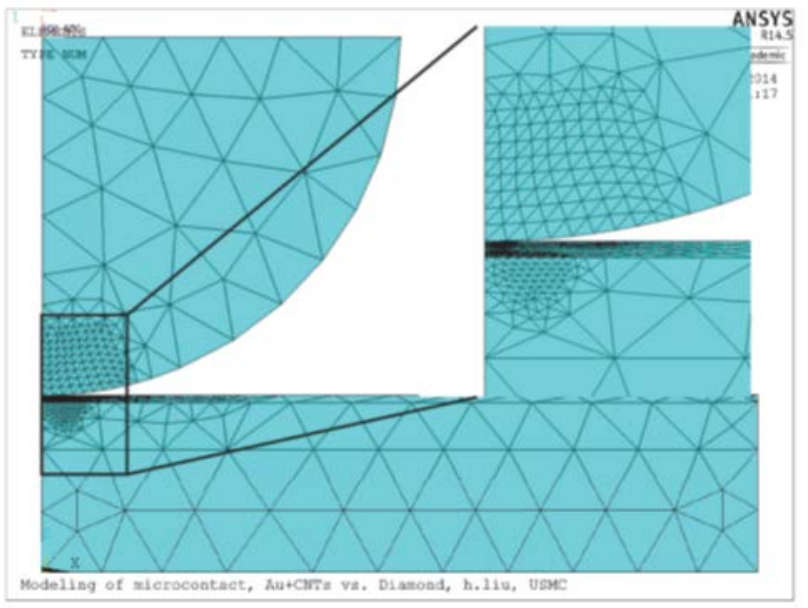

Fig.3 Example of the FEA model used in [4-8].

b)

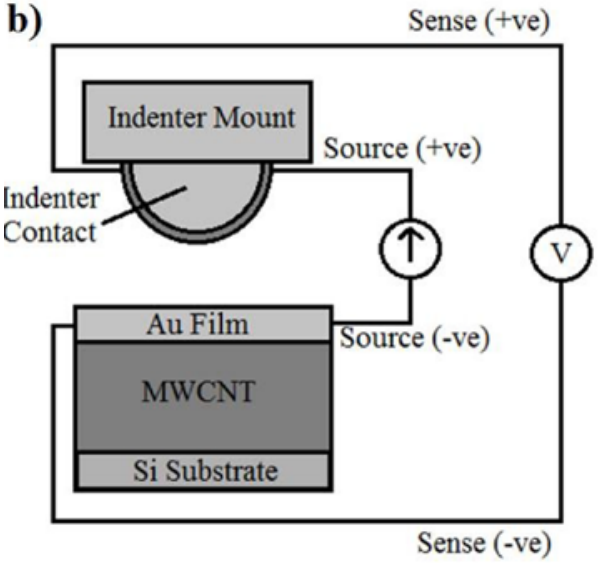

Fig. 4. Nano-indenter experiment to determine constriction resistance.

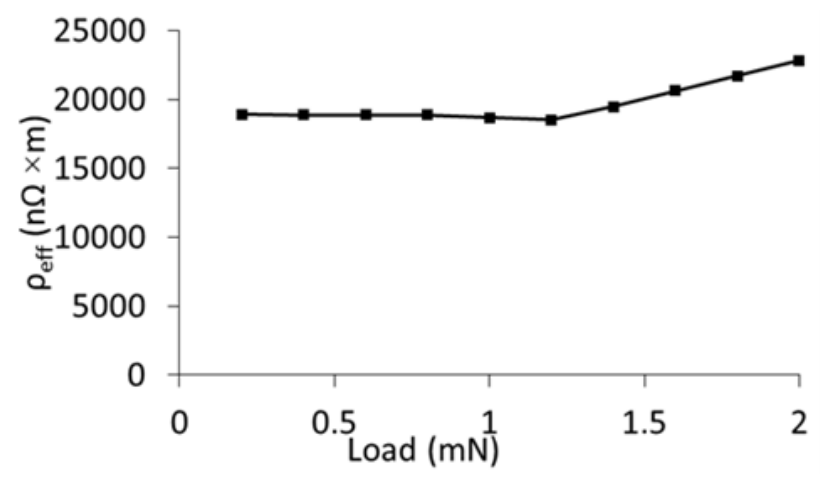

Fig. 5. Effective resistivity, 505 surface, $(<1.2 \mathrm{mN}), \rho_{e}=19400$ n $\Omega . m$. 


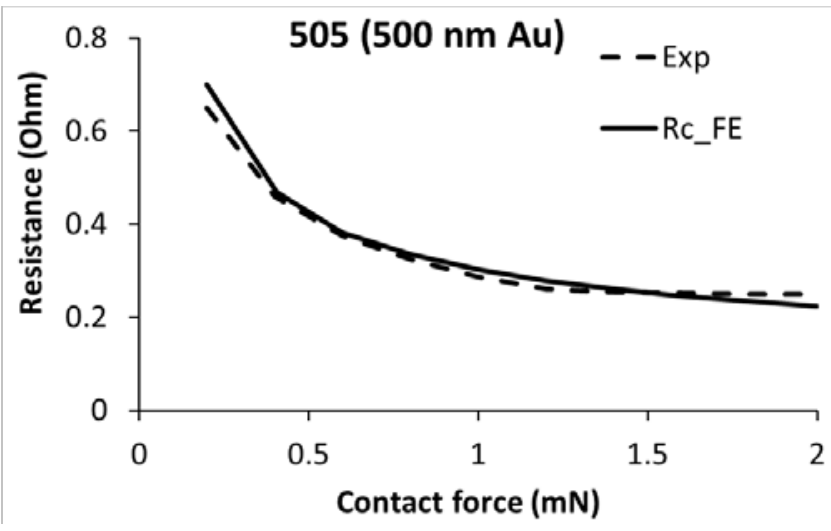

Fig. 6 Updated smooth surface model compared to the experiment data using $\rho_{e}=19400 \mathrm{n} \Omega . \mathrm{m}$

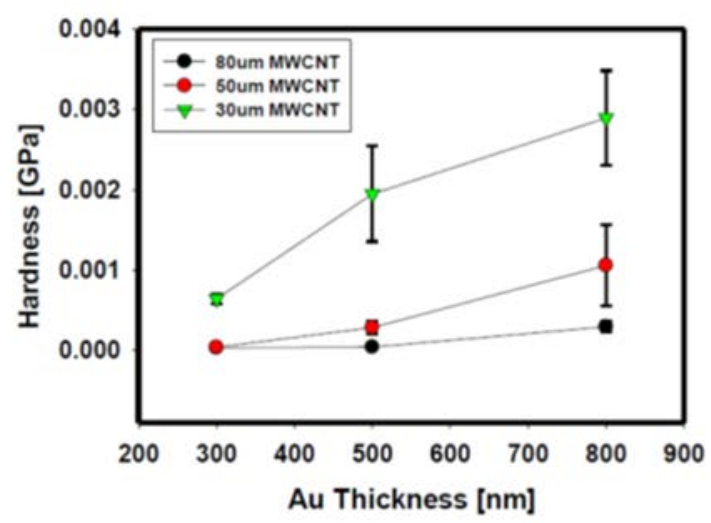

Fig. 7 Nano-indenter Hardness data [11]

\section{B. Experimental reference data.}

A modified nano-indenter was used to for reference, using the system in Fig.4. The data was used to determine the effective resistivity using Eq (1), [9,11]. The effective resistivity for the 505 surface is shown in Fig. 5. It is noted that above $1.2 \mathrm{mN}$ the resistivity increases. In this work we have used a constant value of $\rho_{e}=19400 \mathrm{n} \Omega . \mathrm{m}$.

For a verification of the smooth surface model, a sheet resistivity value was used in [6], with an estimated resistivity of $4378.5 \mathrm{n} \Omega . \mathrm{m}$, or approximately $23 \%$ of the value in Fig $5<1.2 \mathrm{mN}$. The data shown in Fig 6, has been updated here for the effective resistivity in Fig 5 . This shows that the smooth FEA model is highly correlated with the experimental data, although it must be noted that the experimental data is measured on a rough contact surface, as shown in Fig.1. There are two observations, firstly, the application of Eq. (1) assumes a single contact spot and the Hardness value shown in Fig.7, of the deforming bi-layered $\mathrm{Au} / \mathrm{CNT}$ surface; and secondly the Hardness value used is subject to experimental error as shown in Fig.7; although the error bar for the 505 surface $(50 \mu \mathrm{m}$, CNT with a $500 \mathrm{~nm}$ layer of $\mathrm{Au}$ ), shows negligible error.

\section{THE CHARACTERISATION OF ROUGH SURFACES FOR CONTACT AREA DETERMINATION}

A key parameter is all physical contact problems is the real area of contact between rough surfaces, $A$. The determination of $A$ is problematic as the interface between two contacting bodies is difficult to determine. Using measured surface data with "S type" parameters we can consider the link between surface roughness and the real contact area.

\section{A. Surface Roughness using (Sq)}

The $S q$ parameter is the RMS value of the surface amplitude. It is the most basic of the S type parameters and gives a magnitude indication of the surface roughness without reference to the sample length, or the distribution of asperities.

The Au/CNT 505 surface shown in Fig's 1 and 2, can be described as a 3 wavelength surface, as follows; at the smallest wavelength of 1-2 $\mu \mathrm{m}$, the surface is described by features associated with the tips of individual carbon nanotubes, as evidenced in the SEM image in Fig 2. This scale can only be effectively measured with SEM and AFM methods, at this scale the surface exhibits a roughness of, $S q$ $=0.22 \mu \mathrm{m}$, with data resolution of $0.156 \mu \mathrm{m},(\delta=0.156$ $\mu \mathrm{m}),[12]$.

In the next band of wavelength, 2-100 $\mu \mathrm{m}$ we observe the surface shown in Fig, 1, measured with an optical probe with a projected spot size of $5 \mu \mathrm{m}$, using the new XYRIS 2020 L [13], shown with a sample length or data resolution of $0.25 \mu \mathrm{m},(\delta=0.25 \mu \mathrm{m})$. The data is processed using the commercial software (BEX $®)$ [14], to show the standard deviation of the surface, $S q=0.53 \mu \mathrm{m}$. In previous investigations, of similar surfaces, [8], the Sq value has been underestimated (typically $0.2-0.3 \mu \mathrm{m}$ ) as the data was collected with an optical probe with a spot size of $8 \mu \mathrm{m}$. The larger spot size acts like a low pass filter, reducing the peak heights and therefore reducing $S q$.

The third surface wavelength is associated with the waviness of the surface as noted previously $[7,8]$ and in this example is linked to the clumped growth of the CNT surface. This has been shown, characterized by the application of a gaussian filter of wavelength $80 \mu \mathrm{m}$, in [9], on measurements over a $1 \times 1 \mathrm{~mm}$ area with a data resolution of $2 \mu \mathrm{m},(\delta=2 \mu \mathrm{m})$, where the unfiltered surface, $S q=1.5 \mu \mathrm{m}$. Characterizing a true surface roughness $(S q)$ thus depends fundamentally on the measurement sample length $(\delta)$.

The method of computationally generating surface models is described in [8] using MATLAB. The method allows the user to define the $S q$ value for the surface and then uses a correlation function to generate the surface, which can then be imported to the ANSYS FEA model. Typical models are constructed over $1 \mathrm{~mm} \times 1 \mathrm{~mm}$ with a sample length $\delta=2 \mu \mathrm{m}$. To enable the convergence of the FEA model, the sample length is reduced in a further step, using MATLAB code to $\delta=4 \mu \mathrm{m}$.

In this work we assume the surface as described in Fig.1, with a $S q=0.53 \mu \mathrm{m}$. In order to link the rough surface to the contact mechanics of the surface, requires two further parameters, [15], the peak density and the mean summit radius.

\section{B. The Peak Density parameter (Sds) and Mean Summit Curvature (Ssc)}

The summit density $S d s$, is the number of summits per unit area. Summits are derived from peaks, using the ISO standard, [16], and BEX®; a peak is defined as any point, above all 8 nearest neighbors. Summits are constrained to be separated by at least $1 \%$ of the minimum " $\mathrm{X}$ " or "Y" 
dimension comprising the 3D measurement area. Additionally, summits are only found above a threshold that is $5 \%$ of $\mathrm{Sz}$ (maximum peak to valley height) above the mean plane.

Ssc is the Mean Summit Curvature and is the inverse of the mean summit radius. The values are determined from the curvature of the peaks, based on the heights central point and its 4 cardinal neighbors, and assumes a regular sample length. Peaks are found as described above for the summit density. Both parameters are functions of the sample length $(\delta)$ used to make the measurement. The Sds parameter and the associated Ssc are hybrid parameters as they encompass both amplitude and sample length characteristics and provide further details on the functionality of a surface.

Using the MATLAB surface generation method described in [8], with a correlation value (ALC4) generates the surface shown in Fig 8. The generated surface is same area as in the measured data in Fig.1, with the same sample length and $S q$. It is apparent that there is different peak density Sds; for the surface in Fig.8, $S d s=36,355$ peaks $/ \mathrm{mm}^{2}$ while for the measured surface in Fig 1 the value is 193,770 . There are fewer peaks on the simulated surface for the same sample length. An increase in the sample length to $\delta=1 \mu \mathrm{m}$ in the measured data results in a $S d s=$ 65,900 peaks $/ \mathrm{mm}^{2}$, while for the same sample length on the simulated surface $S d s=6,024$ peaks $/ \mathrm{mm}^{2}$. The difference in the peak density is related to the correlation function (ALC4) used, in the MATLAB code.

Increasing the sample length $(\delta$ ) reduces the peak density $S d s$ for the same surface, as fewer peaks are determined. For the ANSYS model surface based on the model shown in Fig. 8 with an increase sample length of $\delta=2 \mu \mathrm{m}$, Sds = 6024, while for $\delta=4 \mu \mathrm{m}$, Sds $=4062$.

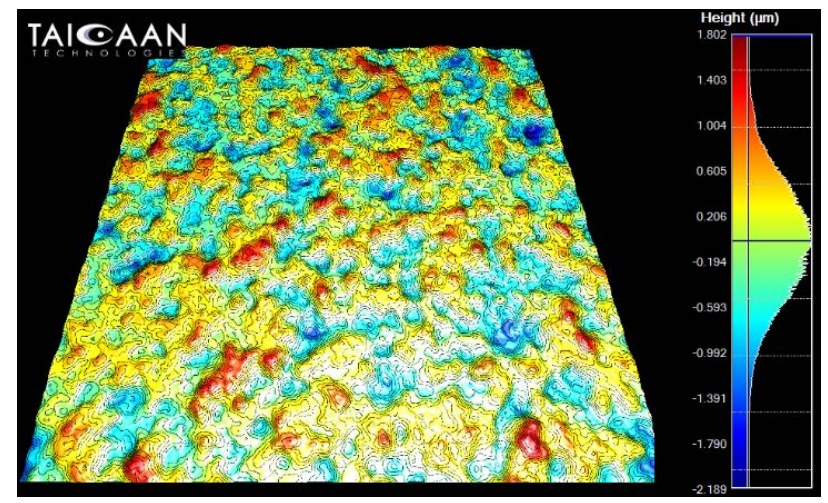

Fig. 8 Model surface $0.15 x 0.15 \mathrm{~mm}, \delta=0.25 \mu \mathrm{m}, S q=0.5 \mu \mathrm{m}$.

\section{Example of output data from the ANSYS code.}

The ANSYS code is designed to determine the contact area $(A)$ and the number of points of contact $(N)$ for the surface imported to the code. With both $A$ and $N$ known we are able to determine $a$ in Eq. 2, and with the effective resistivity we are able to determine the constriction resistance. The surface imported into ANSYS is reduced in area to $0.292 \mathrm{~mm} \times 0.292 \mathrm{~mm}$ and re-sized to $\delta=4 \mu \mathrm{m}$. An output example is shown in Fig.9 for a modelled 505 surface, where the blue area is the rough surface and the lighter areas are the areas of contact at $2 \mathrm{mN}$ force.
The number of contact spots $(N)$ is determined in Matlab, using an image proceeding method, and the total contact area is determined by the ANSYS code, along with the surface displacement and associated parameters.

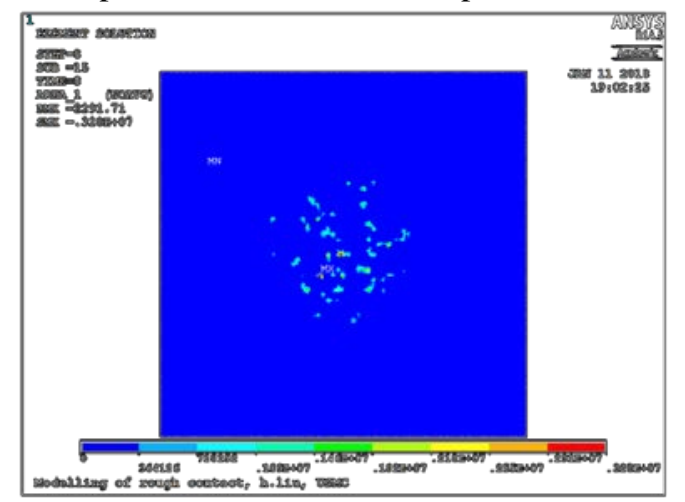

Fig. 9505 model surface at force $2 \mathrm{mN}$ with a $S q$ of $0.5 \mu \mathrm{m}$

\section{Estimation of holm radius and number of contact spots.}

The estimation of the cluster radius (Holm radius), is based on a best fit circle fitting algorithm such that the circle encloses all contact spots in Fig 9. This assumes the surface is nominally flat and rough and without significant waviness, and fits with the underlying physical model of the constriction of the current flow. For the image shown in Fig.9, the circle fitting algorithm provides, $r=62.2 \mu \mathrm{m}$.

\section{MODELLING WORK FLOW}

The full simulation workflow is shown in Fig 10. The first step is to use either the measured surface or the simulated surfaces as described above. In this study we are investigating the surface roughness and as such 7 surfaces of $1 \mathrm{~mm} \times 1 \mathrm{~mm}$ are generated with the same sample length, $\delta=$ $2 \mu \mathrm{m}$, with increasing surface roughness, $S q=0,0.1,0.2$, $0.3,0.4,0.5$, and $1.0 \mu \mathrm{m}$. All samples have the same $S d s=$ 6024. With a constant peak density (Sds) and an increasing roughness standard deviation $(S q)$ the peak summit curvature $(S s c)$ will increase as the average peak radius reduces, as shown in Fig.11.

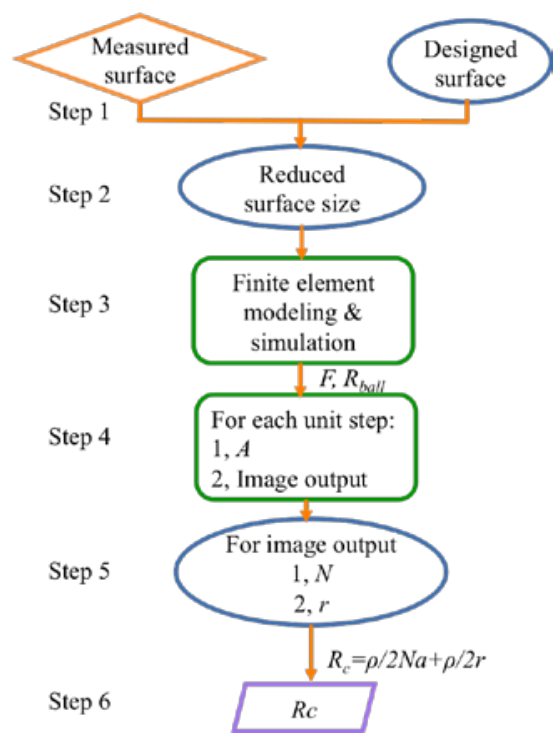

Fig. 10 Workflow for the calculation of constriction resistance 
In the second step the surface is re-sized for the ANSYS simulation convergence criterion, with a reduced area to $0.292 \mathrm{~mm} \times 0.292 \mathrm{~mm}$, with $\delta=4 \mu \mathrm{m}$. The resulting $S d s=$ 4062.

In the third step the ANSYS code is used. To compare with the contact resistance experimental data shown in Fig. 6 , for the 505 surface, the ANSYS analysis is set for 8 force increments, $F=0.25,0.5,0.75,1.0,1.25,1.5,1.75,2 \mathrm{mN}$. The processing time is approximately 24 hours on a desktop environment with 8 core PC with 32GB RAM. The output from the code is step 4 and corresponds to images as shown in Fig. 9. In step 5, each image (e.g. Fig 9) is post processed for $A, a, N$ and $r$ in Eq.2. The final step is the calculation of constriction resistance based on the established value of the effective resistivity.

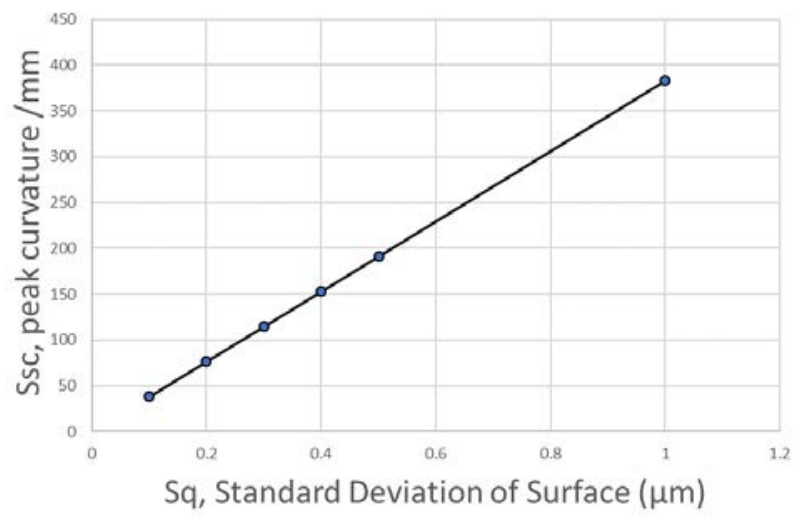

Fig. 11, The linear relationship between peak summit curvature and Sq for a constant Sds (6024) with $\delta=2 \mu \mathrm{m}$.

\section{RESUlts AND DisCUSSION}

Fig. 12 shows a comparison between the experiment result and the FEA model using the $S q=0.5 \mu \mathrm{m}$. It shows a good comparison for the force above $1.5 \mathrm{mN}$. For the low force region, the predicted resistance is between 10-20\% higher than the measured values. For the same model conditions but using a surface with no surface roughness $(S q=0)$ the result of the FEA model from Fig.6 and shown in Fig.10. This shows a close correlation with the experimental result. As discussed above the calculation of the effective resistivity is critical to the resistance and based on the smooth surface single contact point calculation in Eq.1. The difference in the experiment and the FEA data can be assumed to be primary the error associated with the effective resistivity. This suggests that the effective resistivity is $10-20 \%$ lower than the value obtained using Eq.1.

The results in Fig. 12 show the influence of the surface roughness on the contact resistance for a given contact force. The results show that increasing the surface roughness increases the contact resistance up to $S q=0.4$ $\mu \mathrm{m}$. After this level the contact resistance appears to be independent of the roughness of the surface.

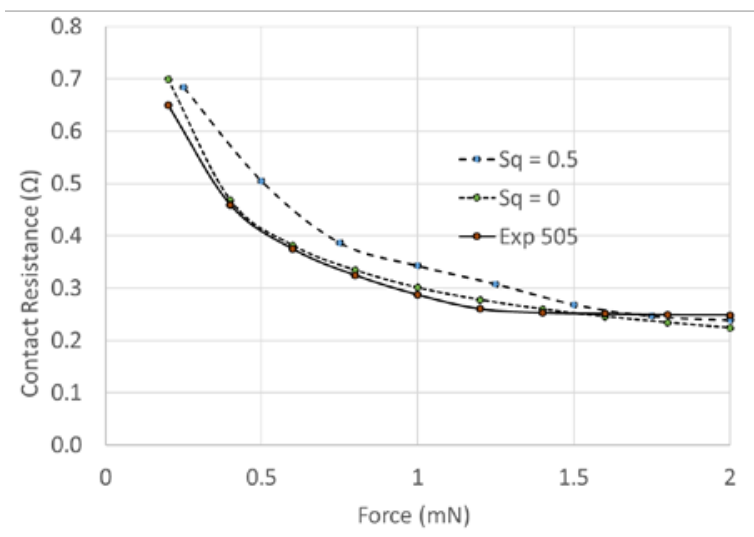

Fig. 12 Verification of the FEA model for a simulated surface

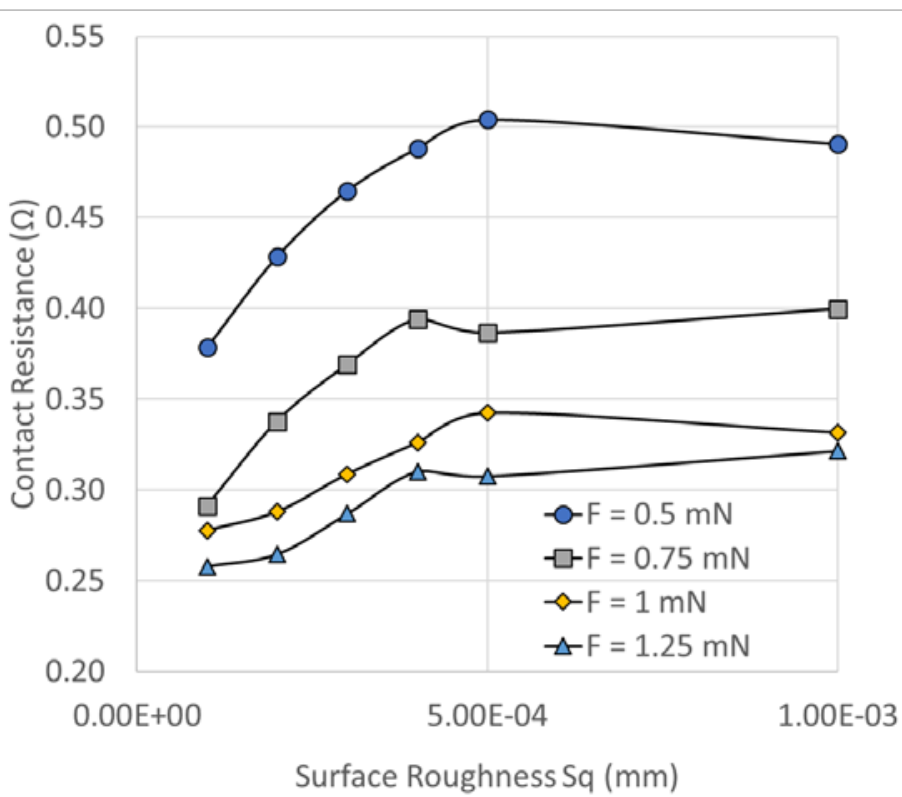

Fig. 13 Variation of Contact Resistance with the Surface roughness, for applied force $(0.5-1.25 \mathrm{mN})$

The verification of the model is based on two assumptions; firstly, the effective resistivity of the surface is constant with force and can be determined from the simple Holm relationship shown in Eq. (1). It is shown that under the conditions investigated, there is an increase in the effective resistivity for higher forces $(>1.2 \mathrm{mN})$ for the rough $\mathrm{Au} / \mathrm{CNT}$ surface (505). Secondly; the contact resistance can be determined from the FEA, contact area results with the application of Eq (2). The results show that the contact resistance from the model is between 10-20\% higher than the experimental value. If we assume that Eq. (2) is the correct model, then there are two possible outcomes.

1. The effective resistivity is overestimated.

2. The RMS $(S q)$ of the modelled surface is too high; increasing the roughness increases the contact resistance (for $\mathrm{Sq}<0.5 \mu \mathrm{m}$ ), as shown in Fig. 13.

\section{A. Error in the Effective Resitivity}

It is important to note that the surface investigated in this study is non-homogenous composite, a mixture that is not uniform in composition and cannot be viewed as a uniform bulk material. It is modelled as a highly elastic under layer 
and an elasto-plastic top layer. The results in [9] have shown that under most compositions of the mixture the effective resistivity is constant, but in this work, re-evaluation the data in [9] for the 505 surface, we have shown that at higher forces $(>1.2 \mathrm{mN})$ it increases with force. It is noted that the estimation of the effective resistivity assumes a single point of contact on a real measured rough surface. All real contact surfaces will have multiple points of contact, as shown in Fig. 9. However, we are unable to determine the unseen measured surface distribution of contact points, although there are some experimental approaches that could be useful in this work. [17].

\section{B. Amplitude Roughness Sq and the contact resistance}

This paper has shown that the $S q$ of the surface along with many other key roughness parameters are fundamental functions of both the sample length used to measure the surface and the sensor type used to make the measurement. The measured value in Fig.1 $(S q=0.5 \mu \mathrm{m})$ has been compared with different sample lengths on the same surface with the same sensor. Because $S q$ is an amplitude parameter it is independent of the sample length, but other parameters such as the number of asperity peaks (Sds) is fundamentally affected.

The results in Fig. 13, show the variation of the surface roughness as defined by the standard deviation (RMS) of the surface area, $(S q)$. These results show that for a given applied contact force that the contact resistance increases with the $S q$ magnitude, up to $0.5 \mu \mathrm{m}$. Above this value the contact resistance is stable. The results show that reducing the $S q$ to $0.1-0.2 \mu \mathrm{m}$, will align the predicted contact resistance with the measured data.

\section{The influence of the the peak summit densiy (Sds)}

The $S d s$ value is important to the distribution of the points of contact. The higher the $S d s$ the more contact points will be in contact with the ball surface. The model surface used in this paper to investigate the effect of roughness changes have shown that the correlation value used in the surface generation model should be lower, the surfaces model have a $S d s$ of $6024(\delta=2 \mu \mathrm{m})$ while the data in Fig. 8 suggest a value of five times higher for the reduced sample length of $\delta=0.25 \mu \mathrm{m}$. We have also shown that the data reduction stage necessary for the FEA model where the sample length is increase further to $\delta=4 \mu \mathrm{m}$, leads to a further reduction of the Sds to 4062.

\section{CONCLUSIONS}

The paper shows that the FEA modelled contact resistance of a Au/CNT surface shows a good alignment with experimental data if the surface is modeled as a smooth surface. The application of a rough surface model increases the contact resistance by a factor of $10-20 \%$. This change has been identified as a factor of the methods used to estimate the effective resistivity of the complex Au/CNT surface.

The paper shows that by using a modeled surface that an increase in the standard deviation (up to $\mathrm{Sq}=0.5 \mu \mathrm{m}$ ) of the rough surface leads to an increase in the contact resistance.

\section{ACKNOWLEDGMENT}

The authors would to thank, Hooi Yee Lim for her development of the image processing methods used in this paper, and Tomas Bull, of TaiCaan technologies for his measurements of the 505 surface using the XYRIS 2020L.

\section{REFERENCES}

[1] E. M. Yunus, S. M. Spearing, and J. W. McBride, 'The Relationship Between Contact Resistance and Contact Force on Au-Coated Carbon Nanotube Surfaces Under Low Force Conditions', IEEE Trans. Compon. Packag. Technol., vol. 32, no. 3, pp. 650-657, Sep. 2009.

[2] B. F. Toler, R. A. Coutu, and J. W. McBride, 'A review of micro-contact physics for microelectromechanical systems (MEMS) metal contact switches,' Journal of Micromechanics and Microengineering, vol. 23, no. 10, Oct, 2013.

[3] J. McBride, 'The wear processes of gold coated multi-walled carbon nanotube surfaces used as electrical contacts for micro-electro-mechanical switching,' Nanoscience and Nanotechnology Letters, 2010, vol. 2, no. 4, pp. 357-361

[4] H. Liu, J. W. McBride, M. P. Down, and S. H. Pu, 'Finite Element Model of a Bilayered Gold-Coated Carbon Nanotube Composite Surface', IEEE Trans. Compon. Packag. Manuf. Technol., vol. 5, no. 6, pp. 779-787, Jun. 2015.

[5] H. Liu and J. W. McBride, 'Finite-Element Contact Modeling of Rough Surfaces Applied to Au-Coated Carbon Nanotube Composites', IEEE Trans. Compon. Packag. Manuf. Technol., vol. 7, no. 3, pp. 329-337, Mar. 2017.

[6] H. Liu and J. W. McBride, 'A Finite Element study of Contact Resistance for a flat bilayered Au/MWCNT surface’, ICEC 2016 UK.

[7] H. Liu, J.W. McBride, 'A Finite Element Based Contact Resistance Model for Rough Surfaces: Applied to a Bilayered Au/MWCNT Composite', IEEE Transactions on Components, Packaging and Manufacturing Technology, 2018, Vol: 8, (6), pp $919-926$

[8] H. Liu and J. W. McBride, 'The influence of multiscale surface roughness on contact mechanics using finite element modelling: applied to a Au-coated CNT composite electrical contact surface', in 2017 IEEE Holm Conference on Electrical Contacts, Denver, CO USA, Sep. 2017, pp. 229235.

[9] M. P. Down, A. P. Lewis, L. Jiang, and J. W. McBride, 'A nano-indentation study of the contact resistance and resistivity of a bi-layered Au/multi-walled carbon nanotube composite', Appl. Phys. Lett., vol. 106, no. 10, p. 101911, Mar. 2015.

[10] Greenwood paper J. A. Greenwood, 'Constriction resistance and the real area of contact,’ Brit. J. Appl. Phys., vol. 17, no. 12, p. 1621, 1966.

[11] M. P. Down, 'Mechanical and Electrical Characterisation of Carbon Nanotube Composite Surfaces for MEMS switching applications,' PhD thesis, University of Southampton, 2015

[12] H. Liu, J. W. McBride, and M. Z. M. Yusop, 'Surface characterization of a Au/CNT composite for a MEMS switching application', in IEEE, Nano 2016, Sendai, Japan, 2016, p. 1-4.

[13] Taicaan Technologies XYRIS 2020L, non contact confocal metrology system, www.taicaan.com

[14] Taicaan Technologies BEX software, www.taicaan.com

[15] J. A. Greenwood, J. B. P. Williamson, 'Contact of Nominally Flat Surfaces’, Proc. R. Soc. Lond. A. 1966, 295, pp 300-319

[16] ISO standard, 25178.

[17] J. W. McBride, K. C. Cross, ‘An Experimental Investigation of the Contact Area between a Glass plane and both Metallic and Carbon Nano-Tube Electrical Contacts', Electrical Contacts, 2008. Proceedings of the 54th IEEE Holm Conference, pp 325 - 331. 